\title{
How do arbidol and its analogs inhibit the SARS-CoV-2?
}

\author{
Aktas $\mathrm{A}^{1}$, Tuzun $\mathrm{B}^{2}$, Taskin $\mathrm{Kafa} \mathrm{AH}^{3}$, Sayin $\mathrm{K}^{2,4}$, Ataseven $\mathrm{H}^{5,6}$ \\ Department of Chemistry, Faculty of Science, Sivas Cumhuriyet University, Sivas, \\ Turkey. krysayin@gmail.com
}

\begin{abstract}
BACKGROUND: COVID-19 is not fully known and causes severe inflammation and cytokine storm. It has many symptoms, such as: fever, sore throat, headache, dyspnoea, and diarrhoea. Arbidol was used in the treatment of COVID19, which was the most critical health problem in the world. However, the desired recovery was not achieved with Arbidol. Many countries still use this drug in the treatment of COVID19. AIM: We aimed to determine whether Arbidol, the hemagglutinin esterase inhibitor used in the treatment of COVID-19, was effective against SARS Cov-2 in silico.

RESULTS AND CONCLUSION: The similarity between hemagglutinin and spike proteins were reported due to the fact that inhibition properties of Arbidol and its 39 analogues were examined in detail against hemagglutinin esterase and spike glycoproteins. CID 1070884 and CID 1207786 were found to be more active against hemagglutinin esterase than in Arbidol, while these compounds were inactive against spike glycoproteins. The interaction mechanism was clarified between arbidol and spike proteins. Phenylalanine, tyrosine, glycine, lysine, and aspartic acid were found to be the headliner amino acids in the interactions between Arbidol and binding domains of spike glycoproteins in the SARS-CoV2 (Tab. 3, Fig. 8, Ref. 28). Text in PDF www.elis.sk

KEY WORDS: SARS-CoV-2, spike glycoprotein, hemagglutinin esterase, arbidol, in silico.
\end{abstract}

\section{Introduction}

The current outbreak of the new coronavirus SARS-CoV2 has spread to many other countries from the Wuhan province of the People's Republic of China. A global health emergency was declared by the World Health Organization (WHO) Emergency Committee on January 30, 2020, due to increased incident reporting rates in China and other areas. Since the beginning of March 2020, while China was carrying a great burden of morbidity and mortality, its incidence was increasing in other Asian countries, Europe, and North America (1).

The most important structural proteins of coronaviruses are trimeric spike $(\mathrm{S})$ protein, membrane $(\mathrm{M})$ protein, envelope $(\mathrm{E})$ protein, and nucleocapsid $(\mathrm{N})$ protein. Beta-Coronaviruses, such as: influenza virus, also have hemagglutinin esterase (HE) glyco-

${ }^{1}$ Department of Internal Medicine, Faculty of Medicine, Sivas Cumhuriyet
University, Sivas, Turkey, ${ }^{2}$ Department of Biochemistry, Faculty of Sci-
ence, Sivas Cumhuriyet University, Sivas, Turkey, ${ }^{3}$ Department of Medical
Microbiology, Faculty of Medicine, Sivas Cumhuriyet University, Sivas,
Turkey, ${ }^{4}$ Advanced Technology Research and Application Centre, Sivas
Cumhuriyet University, Sivas, Turkey, ${ }^{5}$ Department of Gastroenterology,
Faculty of Medicine, Sivas Cumhuriyet University, Sivas, Turkey, and
${ }^{6}$ Ministry of Health of Republic of Turkey, General Directorate of Public
Hospitals, Ankara, Turkey Address for correspondence: K. Sayin, Department of Chemistry, Faculty of Science, Sivas Cumhuriyet University, 58140 Sivas, Turkey.

Acknowledgement: This work is supported by the Scientific Research Project Fund of Sivas Cumhuriyet University under the project number RGD-020. protein. This hemagglutinin esterase enzyme is found in the Coronavirus envelope, more specifically among beta-coronaviruses. Hemagglutinin is an indicator of the evolution of esterase, new Coronavirus, and influenza virus. Hemagglutinin esterase acts as both lectins and receptor-destroying enzymes, mediating reversible binding to O-acetylated-sialic acids $(2,3)$.

For coronavirus 2019 disease (COVID19), which occurs as a result of the coronavirus mutation, scientists are conducting drug studies to treat this disease. In research to date, they have explored more than 30 drugs, including drugs, natural products, and traditional Chinese medicines that may potentially have an effective activity against COVID-19, currently actively used to treat other diseases. Interferon-alpha, lopinavir/ritonavir, ribavirin, chloroquine phosphate, and Arbidol entered the new coronavirus-induced pneumonia treatment guideline by the People's Republic of China National Health Committee (4).

Arbidol is an antiviral drug that was previously approved in China and Russia and used to treat influenza. It acts by preventing the entry and fusion of the virus into the cell by binding to hemagglutinin in influenza (5). There are also studies showing that Arbidol acts in the coronavirus by preventing entry into the cell and fusion. Some studies claim that the Spike (S) protein in coronavirus inhibits the binding of the Angiotensin-Converting Enzyme 2 (ACE2) receptor in humans (6). The hemagglutinin in SARS-CoV2 enables the virus to attach to the cell and enter it. Arbidol reduces the virus's infectivity by clinging to the hemagglutinin and preventing the virus from entering the cell (7). In a study of 69 diseases in China, Arbidol has been shown to reduce the mortality rate and increase healing (8). 


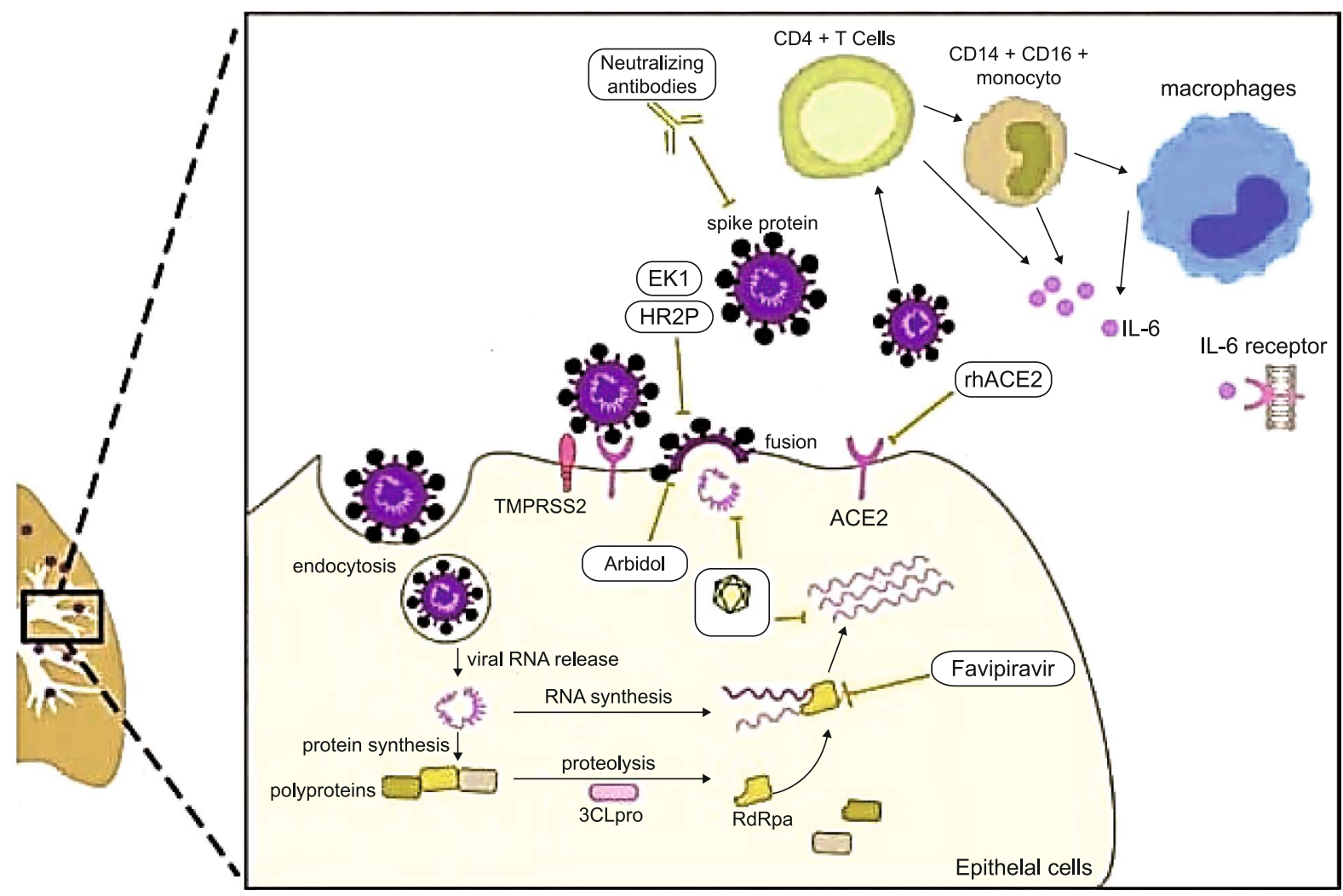

Fig. 1. Used drugs in the treatment of COVID-19 and interaction mechanism of them.

The spike (S) protein found in coronavirus and the Hemagglutinin (HA) protein of Influenza is a member of class I viral membrane fusion proteins. HA and $\mathrm{S}$ protein are multidomain, homotrimeric fusion peptides that act to adhere to the host cell and perform fusion with the host cell membrane. As the result of the evaluations made by cryo-electron microscopy, although $\mathrm{S}$ protein was more complex and significantly larger than HA protein, it was found that the two proteins' structural and functional structure was similar $(9,10)$. Both proteins similarly bind to the sialic acid found in the host cell receptor. Also, HA and S proteins consist of two subunits, H1, H2, and S1, S2, respectively. These functional subunits undergo proteolytic cleavage by the host cell during viral entry into the host cell. This irreversible conformational transition occurring in the protein is believed to be an activating step in fusion (11-13).

This study firstly revealed, how Arbidol interacts with hemagglutinin. Then, the inhibition activity of Arbidol analogues is determined by in silico techniques. Three hundred ninety-five analogues of Arbidol were found in PubChem web page (https:// pubchem.ncbi.nlm.nih.gov/). Arbidol was taken into consideration as reference material, and the full results were compared with the result of Arbidol. As for the target proteins, hemagglutinins belong to the different virus and spike glycoprotein of SARS Coronavirus were taken into consideration. All proteins were selected from protein data bank web tool (https://www.rcsb.org/). In this stage, six proteins, 4WSS, 4WSU, 4WSV, 5XL3, 5XL9, and 5XLA (14,
15), were selected for the determination of active drug candidate. The interaction mechanism of drugs and Arbidol used in the treatment of COVID-2019 is shown in Figure S1 (16).

Then, ligand-receptor interactions of active compounds and Arbidol was examined in detail. In this step, the receptor was selected as 6VSB and 6VYB $(17,18)$. The interaction mechanism between Arbidol and spike glycoprotein of SARS-CoV2

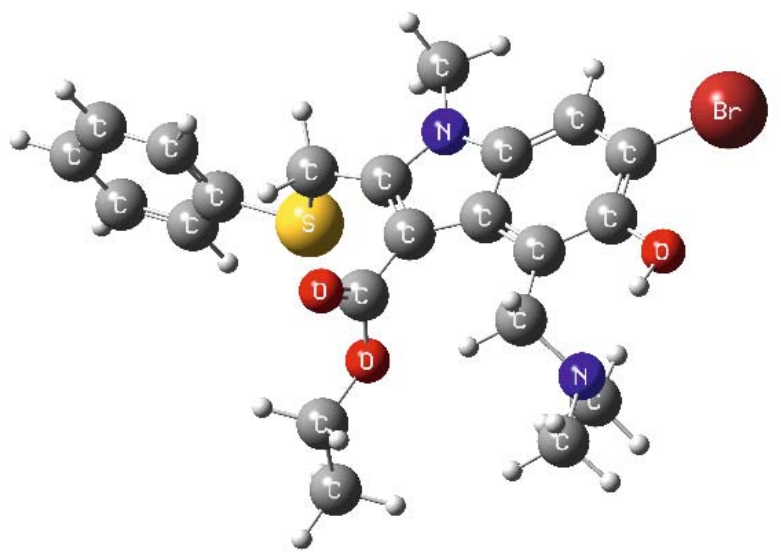

Fig. 2. Structure of ethyl 6-bromo-4-[(dimethylamino)methyl]-5-hydroxy-1-methyl-2-(phenylsulfanylmethyl)indole-3-carboxylate, which is Arbidol with atomic symbol labels. 
Tab. 1. The ligands ${ }^{a}$ interacted with the target proteins.

\begin{tabular}{lllllll}
\hline Compound ID & 4WSS & 4WSU & 4WSV & 5XL3 & 5XL9 & 5XLA \\
\hline 131411 & OS, PS & OS, PS & OS, PS & OS, PS & OS, PS & OS, PS \\
629708 & OS & OS & OS & OS & OS & OS \\
981133 & OS, PS & OS, PS & OS, PS & OS, 2xPS & OS & OS \\
1070875 & OS, PS & OS, PS & OS & OS, PS & OS, PS & OS, PS \\
1070876 & OS & OS & OS & OS & OS & OS \\
1070877 & OS & OS, PS & OS & OS & OS & OS \\
1070881 & OS, PS & OS, PS & OS & OS, PS & OS, PS & OS, PS \\
1070882 & OS, PS & OS, PS & OS, PS & OS, PS & OS, PS & OS, PS \\
1070884 & OS, PS & OS, PS & OS, PS & OS, PS & OS, PS & OS, PS \\
1124434 & OS & OS, PS & OS & OS & OS & OS \\
1124440 & OS & OS & OS & OS & OS & OS \\
1124443 & OS, PS & OS, 2xPS & OS, PS & OS, 3xPS & OS & OS \\
1124444 & OS, PS & OS & OS, PS & OS, PS & OS, PS & OS, PS \\
1201931 & OS, $2 x P S$ & OS, 3xPS & OS, $3 \times P S$ & OS, 2xPS & OS, 2xPS & OS, 2xPS \\
1207786 & OS, PS & OS, PS & OS, PS & OS, PS & OS, PS & OS, PS \\
1238513 & OS, PS & OS, PS & OS, PS & OS, PS & OS, PS & OS, PS \\
1238708 & OS, PS & OS, 2xPS & OS, PS & OS & OS, PS & OS, PS \\
1239099 & OS & OS, PS & OS & OS & OS, PS & OS \\
1252264 & OS & OS & OS & OS & OS & OS \\
1253698 & OS & OS & OS, PS & OS & OS, PS & OS \\
1253892 & OS, 2xPS & OS, PS & OS, 2xPS & OS, PS & OS, 2xPS & OS, PS \\
1283486 & OS, PS & OS, PS & OS, PS & OS, 2xPS & OS, PS & OS, PS \\
1502917 & - & - & OS & - & - \\
\hline
\end{tabular}

${ }^{\text {a }}$ OS: Original Structure; PS: Possible Structure, ${ }^{\text {b }}$ CID 131411 is arbidol

was examined in detail. Then, the inhibition efficiencies of active compounds were compared with the efficiency of Arbidol. So, more active compounds than that of Arbidol were suggested as the most potent drug candidate. Finally, "absorption, distribution, metabolism, and excretion" (ADME) analyses of a preferred drug candidate(s) were performed in detail.

\section{Methods}

Arbidol and its 395 analogous were taken from PubChem. The whole compounds minimized at the OPLS3e method at $\mathrm{pH}=5 \pm$ 4. The possible states of investigated compounds at different $\mathrm{pH}$ were determined. As for the target proteins, hemagglutinin proteins, which belong to the different virus, for instance, influenza, etc. were taken into consideration to specify the active compounds. Selected target proteins were 4WSS, 4WSU, 4WSV, 5XL3, 5XL9, and $5 \mathrm{XLA}(16,17)$. It was then determined, which active compounds would inhibit the spike glycoprotein of the SARS coronavirus, which were 6VSB and 6VYB (19,2 0). Before docking calculations, target proteins were minimized at the OPLS3e method at $\mathrm{pH}=5 \pm 4$. Then, active regions of $4 \mathrm{WSS}$, 4WSU, 4WSV, 5XL3, 5XL9, and 5XLA were determined by using reported data. As for the spike glycoprotein, active sites of 6VSB and 6VYB were determined by the calculation of Site Map (21-23). How Arbidol inhibited the spike protein of SARS coronavirus was explained in detail. As for the determination of target small molecules as a drug candidate, the whole active compounds were docked with 6VSB and 6VYB proteins. The results of active compounds were compared with the results of Arbidol. All calculations were performed with Schrödinger / Maestro 12.2 program (24-29).

\section{Results and discussion}

Arbidol and its analogues as drug candidate

Arbidol (PubChem ID: CID 131411), represented in Figure 2 and its analogues, were taken into consideration. PubChem ID and International Union of Pure and Applied Chemistry (IUPAC) names of Arbidol's analogues are given in Supp Table 1.

Arbidol and all selected compounds were optimized at the OPLS3e method at $\mathrm{pH}=5 \pm 4$. The possible structures at different

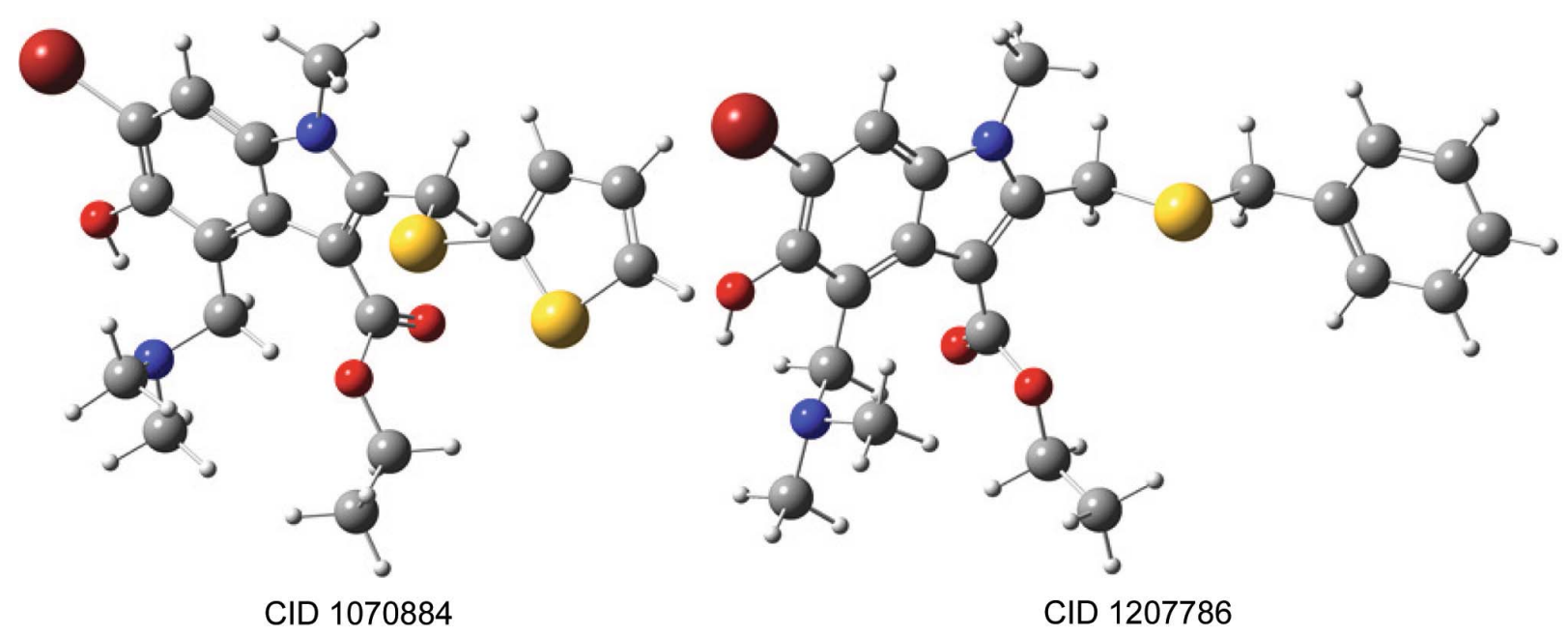

Fig. 3. The structure of CID 1070884 and CID 1207786. 

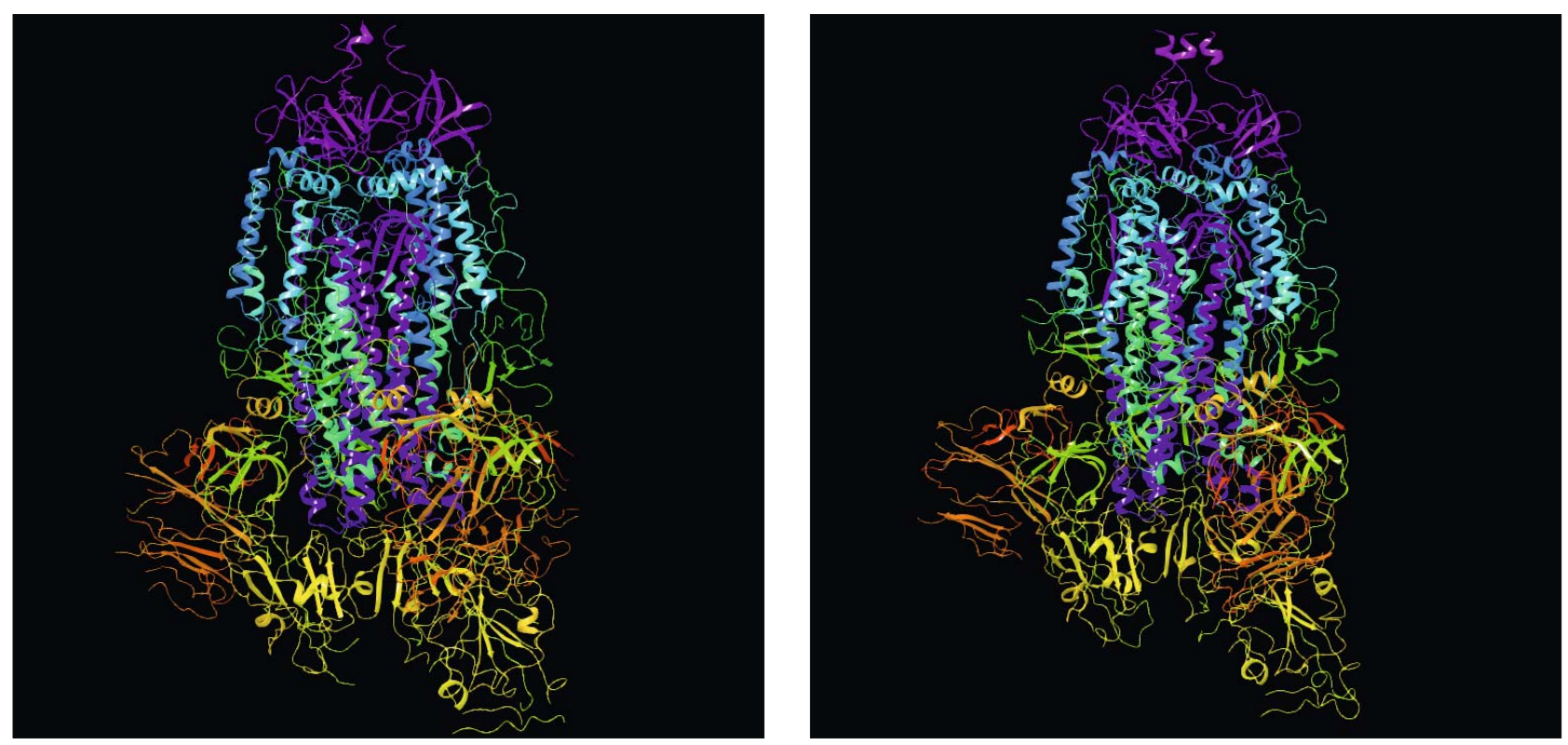

Fig. 4. The protein structures of 6VSB (on the left) and 6VYB (on the right).

$\mathrm{pH}$ levels were determined for each compound. As the result, 1684 possible structures were found. The whole original structure and possible structures of studied compounds were taken into consideration in the docking calculations at hemagglutinin target proteins.

\section{Docking analyses with hemagglutinin proteins}

Arbidol and its analogues interacted with the target hemagglutinin proteins by the molecular docking calculations. Before the calculations, target proteins were minimized at the OPLS3e method. Active regions were defined by using the published article. Arbidol and the whole possible structures tried to interact with these proteins. According to the obtained results, it was found that most of the selected compounds were inactive against the hemagglutinin proteins. The active compounds are given in Table 1. Additionally, the docking structure and interaction of Arbidol are represented in Figure 3.

According to Table 1, only 22 Arbidol analogues were active against hemagglutinin. Arbidol and one of the possible structures inhibited each target protein. Some drug candidates were deter-

Tab. 2. Molecular docking results.

\begin{tabular}{lcccc}
\hline Compound ID & $\mathrm{a}^{-D S}{ }^{\mathrm{a}}$ & $\mathrm{a}-\mathrm{E}_{\mathrm{vdw}}{ }^{\mathrm{a}}$ & $\mathrm{a}_{\mathrm{Coul}}{ }^{\mathrm{a}}$ & ${\mathrm{a}-\mathrm{E}_{\text {Total }}{ }^{\mathrm{a}}}$ \\
\hline 131411 (Arbidol) & -1.963 & -29.935 & -5.414 & -35.349 \\
1070882 & -2.007 & -29.506 & -4.887 & -34.393 \\
1070884 & -2.244 & -28.760 & -6.682 & -35.442 \\
1124443 & -2.180 & -22.324 & -10.077 & -32.401 \\
1201931 & -1.613 & -26.730 & -6.419 & -33.149 \\
1207786 & -2.153 & -28.830 & -7.367 & -36.197 \\
1238513 & -2.066 & -25.815 & -5.801 & -31.617 \\
1238708 & -2.043 & -25.184 & -7.873 & -33.056 \\
1253892 & -1.894 & -28.203 & -7.292 & -35.495 \\
1283486 & -1.897 & -27.941 & -7.473 & -35.414 \\
\hline
\end{tabular}

a in $\mathrm{kcal} / \mathrm{mol}$ mined more active than two structures for each protein, while others less than two. When we advanced the analysis in this way, it was determined that the inhibition activation of CID 1070882, CID 1070884, CID 1124443, CID 1201931, CID 1207786, CID 1238513, CID 1238708, CID 1253892 and CID 1283486 were found similar to Arbidol or more than that of Arbidol. The average docking score (a-DS), average van der Waals energy ( $a-E_{v d w}$ ), average coulomb energy $\left(\mathrm{a}-\mathrm{E}_{\mathrm{Cou}}\right)$, and average total interaction energy $\left(\mathrm{a}-\mathrm{E}_{\text {Total }}\right)$ of Arbidol and active compounds are given Table 2.

Tab. 3. Molecular docking results of Arbidol with active sites of the target proteins.

\begin{tabular}{ccccc}
\hline Active Site & $\mathrm{DS}^{\mathrm{a}}$ & $\mathrm{E}_{\mathrm{vdw}}{ }^{\mathrm{a}}$ & $\mathrm{E}_{\text {Coul }}{ }^{\mathrm{a}}$ & $\mathrm{E}_{\text {Total }}{ }^{\mathrm{a}}$ \\
\hline $6 \mathrm{VSB}$ & & & & \\
1 & -6.003 & -37.025 & -2.740 & -39.765 \\
2 & -3.349 & -36.039 & -2.320 & -38.359 \\
3 & -4.577 & -33.809 & -8.600 & -42.409 \\
4 & -5.780 & -42.516 & -5.568 & -48.084 \\
5 & -5.811 & -36.703 & -6.694 & -43.397 \\
6 & -4.414 & -38.374 & -1.510 & -39.883 \\
7 & -3.710 & -35.074 & -3.338 & -38.413 \\
8 & -5.441 & -32.513 & -4.894 & -37.407 \\
9 & -4.798 & -39.746 & -6.162 & -45.908 \\
10 & -5.789 & -34.845 & -7.587 & -42.432 \\
\hline $6 \mathrm{VYB}$ & & & & \\
1 & -3.961 & -36.548 & -4.438 & -40.986 \\
2 & -5.120 & -35.616 & -7.069 & -42.685 \\
3 & -2.637 & -32.727 & -6.415 & -39.142 \\
4 & -3.806 & -30.219 & -8.405 & -38.624 \\
5 & -3.247 & -30.134 & -8.390 & -38.524 \\
6 & -3.758 & -43.268 & -2.336 & -45.603 \\
7 & -2.806 & -29.547 & -1.030 & -30.578 \\
8 & -2.860 & -37.575 & -4.794 & -42.369 \\
9 & -6.882 & -35.028 & -3.880 & -38.908 \\
10 & -3.935 & -36.990 & -6.402 & -43.392 \\
\hline a in kcal/mol & & & & \\
& & & &
\end{tabular}



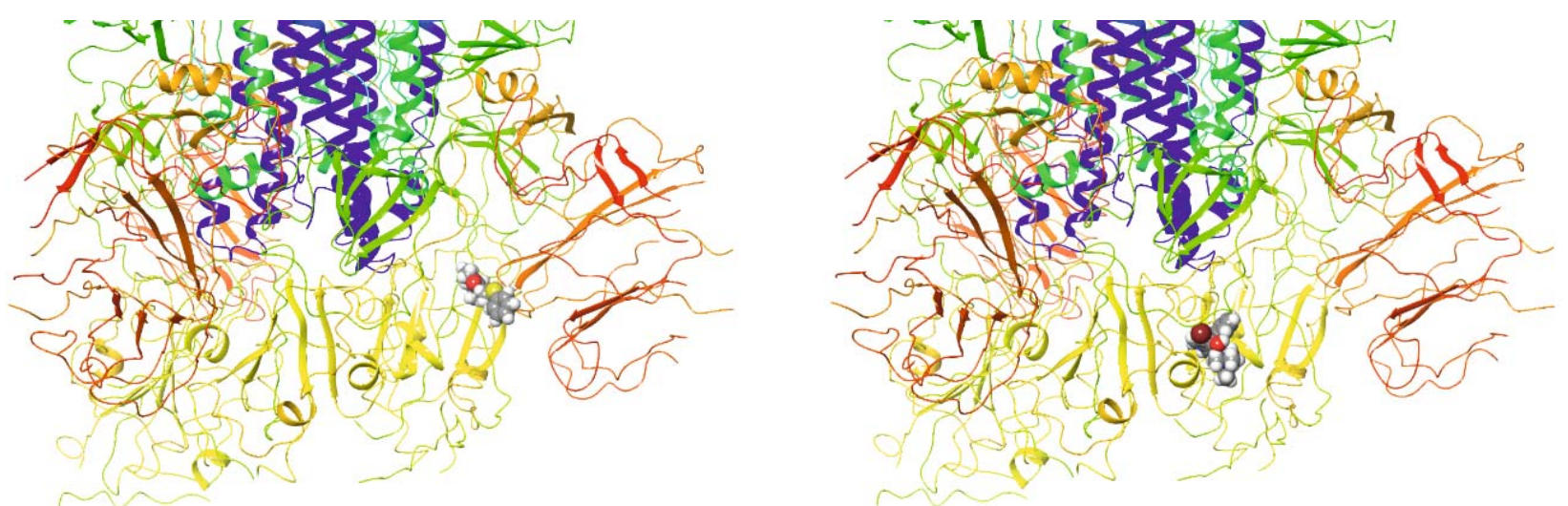

Fig. 5. The complex structure at active site 7 (on the left side) and 8 (on the right side) in 6VSB.
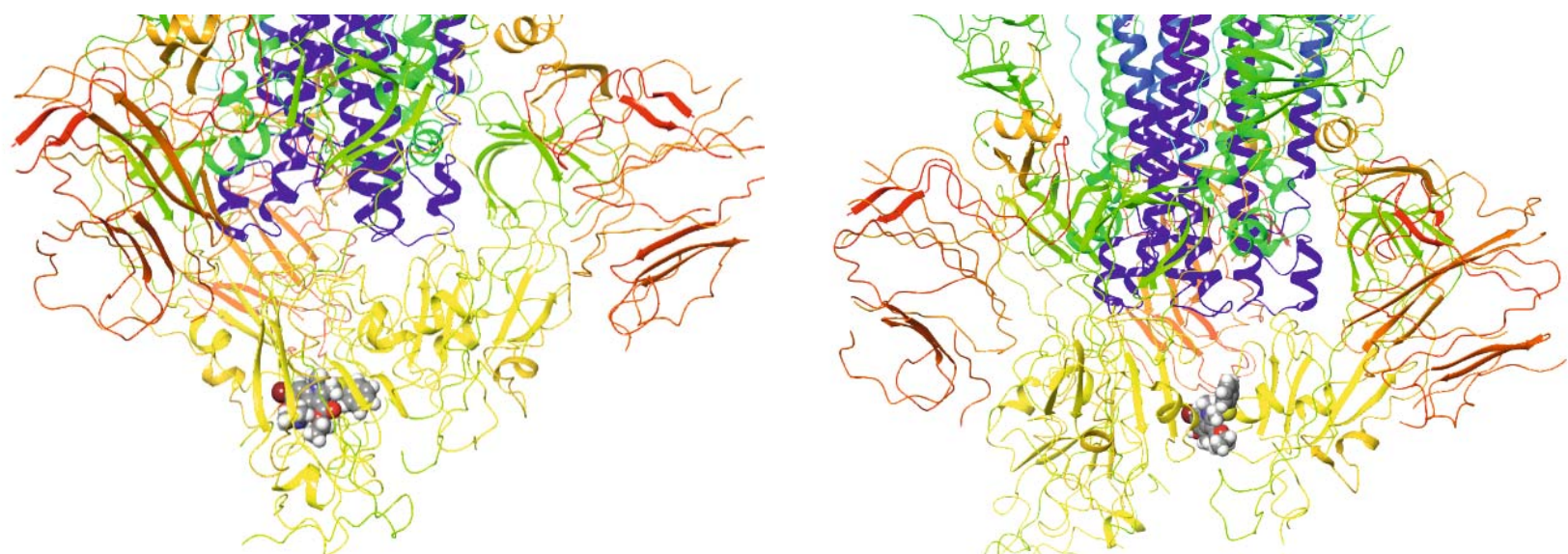

Fig. 6. The complex structure at active site 9 (on the left side) and 10 (on the right side) in 6VYB.

According to Table 2, the requested results were better in docking score and better in total interaction energy than Arbidol results. In this study we focused on finding the best drug candidates. As the results, two compounds, which were CID 1070884 and CID 1207786 attracted the attention, whose IUPAC names were Ethyl 6-bromo-4-[(dimethylamino)methyl]-5-hydroxy-1-methyl2-(thiophene-2-ylsulfanylmethyl)indole-3-carboxylate and Ethyl 2-(benzylsulfanylmethyl)-6-bromo-4-[(dimethylamino)methyl]5-hydroxy-1-methylindole-3-carboxylate, respectively. Because all the results except the average van der Waals energy of them were better than that of Arbidol. These compounds are represented in Fig. 3 and were taken into consideration for further analyses.

Molecular docking analyses of arbidol and its active analogues

The significant suspicion about COVID-19 is whether there is hemagglutinin protein. Hemagglutinin and spike glycoprotein play an essential role in adhering to the host cell. Furthermore, the similarity between hemagglutinin and spike proteins were explained in detail in the introduction section. As for the other perspective, Arbidol inhibits the hemagglutinin esterase. We think that Arbidol inhibits the spike glycoprotein of SARS-CoV2 due to the similarity between $\mathrm{S}$ and HE proteins. The SARS-CoV2 S proteins were selected from the protein data bank as 6VSB and $6 \mathrm{VYB}$, represented in Figure 4. These proteins were minimized at the OPLS3e method, and active sites were determined. The active sites are represented in Supp. Fig. S1 - S10.

$\mathrm{S}$ domain of spike glycoprotein of the SARS-CoV2 virus plays the vital role in the binding of the virus to human ACE2. Therefore, these domains are the target for the inhibition of the spike glycoproteins of SARS-CoV2. If these domains can be inhibited by any compounds, the virus, SARS-CoV2, will block the spread of the human body. These domains encounter active sites 7 and 8 for $6 \mathrm{VSB}$ protein; active sites 9 and 10 for $6 \mathrm{VYB}$ protein. These sites were determined as target points in this study. The docking calculations were performed with Arbidol and each active site. The docking score (DS), van der Waals energy $\left(\mathrm{E}_{\mathrm{vdw}}\right)$, Coulomb energy $\left(\mathrm{E}_{\mathrm{Coul}}\right)$, and total interaction energy $\left(\mathrm{E}_{\text {Total }}\right)$ are given in Table 3.

According to Table 3, Arbidol binds the whole active sites in target proteins. This is the negative result of Arbidol. Because the amount of Arbidol in the human body decreases with the inhibition of other active sites, so Arbidol does not exhibite the desired activity. However, this result does not imply that Arbidol is inactive against spike proteins. As for the docking results, the docking score, related to the key-lock harmony between ligand and recep- 

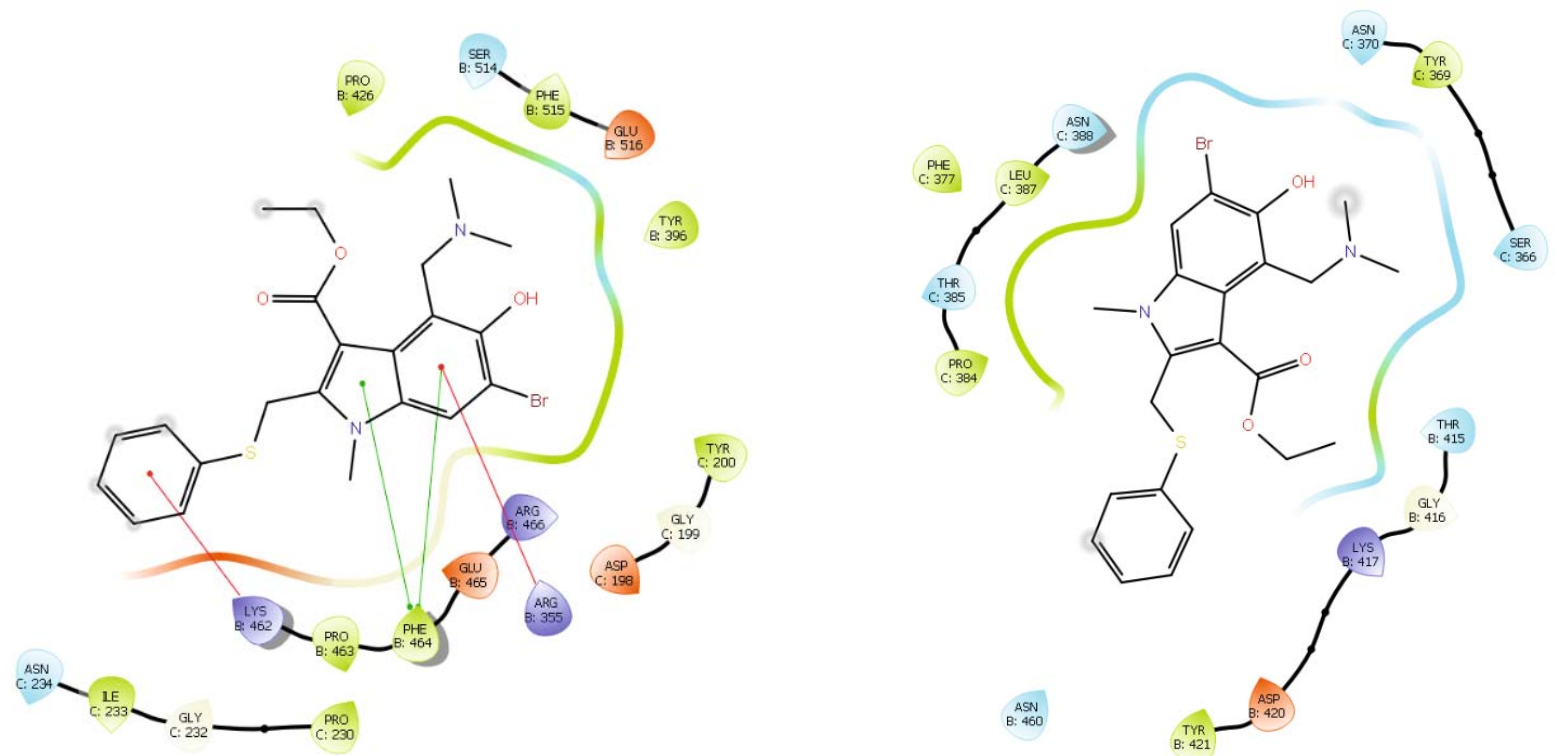

Fig. 7. The interaction schema at active site 7 (on the left side) and 8 (on the right side) in 6VSB.
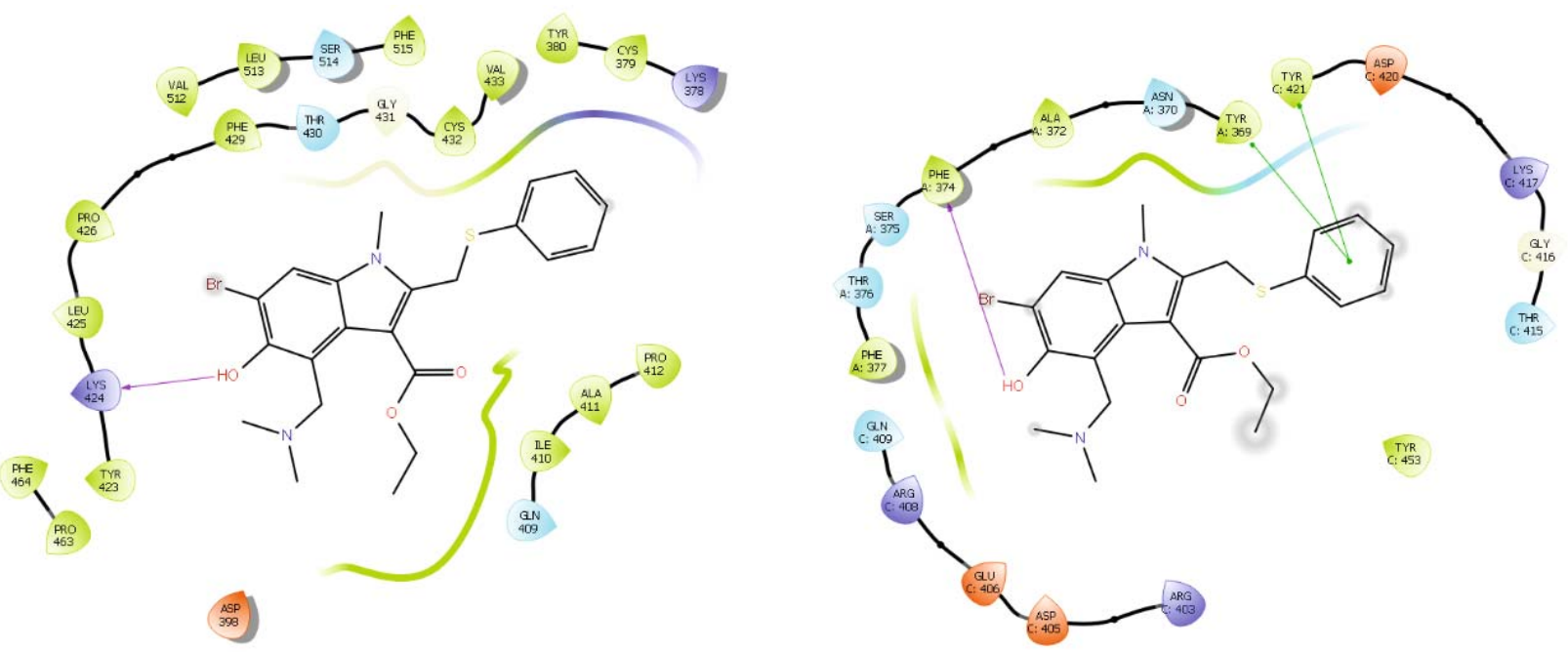

Fig. 8. The interaction schema at active site 9 (on the left side) and 10 (on the right side) in 6VYB.

tor, it was calculated as better at active site 1 in $6 \mathrm{VSB}$ and active site 9 in 6VYB. The charge-charge (physical) interactions were lower than those of van der Waals interactions. The total interaction energy was determined as better at active site 9 in 6VSB and active site 10 at $6 \mathrm{VYB}$. Although the results seem confusing, the docking score and interaction energy were good. So, good docking score or good total interaction energy does not mean it is useful. It would be more logical to choose interactions, where both data are right. If the results are reviewed again, it can be said that Arbidol probably binds more to active site 4 in 6VSB and active site in 6VYB. Looking at published clinical cases, we reported why the effect of Arbidol was lower than other drugs. As for the interaction between the Arbidol and binding domain of spike glycoprotein, ligand-receptor complex structures for 6VSB and 6VYB are represented in Figures 5 and 6, respectively.
Arbidol inhibits the binding domains in each target protein. Additionally, interaction schemas for the above interaction in 6VSB and 6VYB are represented in Figures 7 and 8, respectively.

According to Figures 7 and 8, the observed interaction types are hydrophobic, negative charged, positively charged, glycine, polar, solvent exposure, pi-pi stacking, pi-cation interactions, and hydrogen bond. Negative and positive charged are summed in coulomb interactions energies, while others form the van der Waals energy. Additionally, phenylalanine, tyrosine, glycine, lysine, and aspartic acid are headliner amino acids in the interaction between the Arbidol and binding domain of spike glycoproteins in the SARS-CoV2.

As for the active compounds, which are CID 1070884 and CID 1207786, molecular docking calculations between selected active compounds and binding domain of target proteins were performed. However, it was found that these compounds were 
inactive against the binding domain. Furthermore, the interaction between active compounds and active sites in each protein found that the selected compounds were inactive against spike glycoprotein. As the result, it can be said that inhibition properties of CID 1070884 and CID 1207786 against hemagglutinin esterase were better than that of Arbidol, while these compounds were inactive against spike glycoproteins of SARS-CoV2 virus.

\section{Conclusion}

The biological activity in the inhibition of hemagglutinin esterase and spike glycoprotein of Arbidol and its analogues were investigated by the in silico technique. Initially, Arbidol and its analogues were minimized at the OPLS3e method at $\mathrm{pH}=5 \pm 4$. 4WSS, 4WSU, 4WSV, 5XL3, 5XL9, and 5XLA were selected as hemagglutinin esterase, while 6VSB and 6VYB were selected as spike glycoprotein from the protein data bank. Molecular docking calculations were performed with the related compounds and hemagglutinin proteins. In the calculation results, CID 1070884 and CID 1207786 were found to be more active than those of Arbidol against hemagglutinin esterase proteins. The interaction mechanism of Arbidol was examined in detail against spike glycoprotein of the SARS-CoV2 virus. It was reported, why the effect of Arbidol was lower than the other clinical drugs. Phenylalanine, tyrosine, glycine, lysine and aspartic acid were found as the headliner amino acids in the interactions between Arbidol and binding domain of spike glycoproteins in the SARS-CoV2. Finally, it was found that arbidol analogues were inactive against the spike glycoproteins.

\section{References}

1. Dong E, Du H, Gardner L. An interactive web-based dashboard to track COVID-19 in real time. The Lancet 2020. DOI: 10.1016/S14733099(20)30120-1.

2. Lu R, Zhao X, Li J et al. Genomic characterisation and epidemiology of 2019 novel coronavirus: implications for virus origins and receptor binding. The Lancet 2020; 395 (10224): 565-574.

3. Carlos WG, Dela Cruz CS, Cao B et al. Novel Wuhan (2019-nCoV) coronavirus. Am J Respir Crit Care Med 2020; 201: 7-8.

4. Paules CI, Marston HD, Fauci AS. Coronavirus infections-More than just the common cold. JAMA 2020; 323 (8): 707-708.

5. Prajabat M, Sarma P, Shekhar N et al. Drug targets for Coronavirüs: A sistematic review. Indian J Pharmacol 2020; 52: 56-65.

6. Dong L, Hu S, Gao J. Discovery drugs to treat coronavirus disease 2019 (COVID-19). Drug Discoveries Therapeutics 2019; 14 (1): 58-60.

7. Wu C, Liu Y, Yang Y et al. Analysis of therapeutic targets for SARS$\mathrm{CoV}-2$ and discovery of potential drugs by computational methods. Acta Pharm Sinica B 2020. DOI: 10.1016/j.apsb.2020.02.008.

8. Song Y, Zhang M, Yin L et al. COVID-19 Treatment: Close to a Cure? - A Rapid Review of Pharmacotherapies for the Novel Coronavirus. Preprints 2020; 2020030378.

9. Duan L, Zhu G. Psychological interventions for people affected by the COVID-19 epidemic. The Lancet Psychiatry 2020; 7 (4): 300-302.
10. Wang Z, Yang B, Li Q et al. Clinical Features of 69 Cases with Coronavirus Disease 2019 in Wuhan, China. Clin Infect Dise 2020. doi:10.1093/ cid/ciaa272.

11. Li F. Structure, Function, and Evolution of Coronavirus Spike Proteins. Ann Rev Virol 2016; 3 (1): 237-261.

12. Wilson IA, Skehel JJ, Wiley DC. Structure of the haemagglutinin membrane glycoprotein of influenza virus at $3 \AA$ resolution. Nature 1981; 289: 366-373.

13. Belouzard S, Chu VC, Whittaker GR. Activation of the SARS coronavirus spike protein via sequential proteolytic cleavage at two distinct sites. Proc Natl Acad Sci USA 2009; 106 (14): 5871-5876.

14. Tortorici MA, Walls AC, Lang $Y$ et al. Structural basis for human coronavirus attachment to sialic acid receptors. Nat Struct Mol Biol 2019; 26 (6): 481-489.

15. Devaux CA, Rolain J-M, Colson $P$ et al. New insights on the antiviral effects of chloroquine against coro- navirus: what to expect for COVID-19? Internat J Antimicrob Agents 2020. DOI: 10.1016/j.ijantimicag.2020.105938.

16. Yang H, Carney PJ, Chang JC et al. Structure and Receptor Binding Preferences of Recombinant Hemagglutinins from Avian and Human H6 and H10 Influenza A Virus Subtypes. J Virol 2015; 89: 4612-4623.

17. Song H, Qi J, Xiao $\mathbf{H}$ et al. Avian-to-Human Receptor-Binding Adaptation by Influenza A Virus Hemagglutinin H4. Cell Reports 2017; 20 (5): 1201-1214.

18. Li H, Zhou Y, Zhang M et al. Updated approaches against SARSCoV-2. Antimicrob Agents Chmeother 2020. DOI: 10.1128/AAC.0048320.

19. Wrapp D, Wang N, Corbett KS et al. Cryo-EM structure of the 2019$n C o V$ spike in the prefusion conformation. Science 2020; 367: 1260-1263.

20. Walls AC, Park YJ, Tortorici MA et al. Structure, Function, and Antigenicity of the SARS-CoV-2 Spike Glycoprotein. Cell 2020; 181 (2): 281-292.e6.

21. Halgren T, New method for fast and accurate binding-site identification and analysis. Chem Biol Drug Des 2007; 69: 146-148.

22. Halgren T. Identifying and characterizing binding sites and assessing druggability. J Chem Inf Model 2009; 49: 377-389.

23. Schrödinger Release 2019-4: SiteMap, Schrödinger, LLC, New York, NY, 2019.

24. Harder E, Damm W, Maple J et al. OPLS3: a force field providing broad coverage of drug-like small molecules and proteins, Journal of chemical theory and computation 2016; 12 (1): 281-296.

25. Friesner RA, Murphy RB, Repasky MP et al. Extra precision glide: Docking and scoring incorporating a model of hydrophobic enclosure for protein-ligand complexes. J Med Chem 2006; 49 (21): 6177-6196.

26. Friesner RA, Banks JL, Murphy RB et al. Glide: a new approach for rapid, accurate docking and scoring. 1. Method and assessment of docking accuracy. J Med Chem 2004; 47 (7): 1739-1749.

27. Schrödinger Release 2019-4: LigPrep, Schrödinger, LLC, New York, NY, 2019.

28. Schrödinger Release 2019-4: Maestro, Schrödinger, LLC, New York, NY, 2019.

Received May 20, 2020. Accepted June 1, 2020. 